\title{
Lesiones medulares catastróficas en el rugby argentino. Impacto de las medidas implementadas y su reducción relativa en el tiempo
}

\author{
Federico Carpani," Fernando Salvat,, Marcelo Saco," Nicole Farcy,, Carolina Zamorano," Verónica Bruno,"\# \\ Mauricio F. Farez, "Martín Nogués* \\ *Departamento de Neurología, Fleni, Ciudad Autónoma de Buenos Aires, Argentina \\ ** Departamento Médico, Unión Argentina de Rugby (UAR), Buenos Aires, Argentina \\ ${ }^{*}$ CEBES, Centro de Epidemiología, Bioestadística y Salud Pública, Buenos Aires, Argentina \\ \#\#Departamento de Neurociencias Clínicas, Universidad de Calgary, Calgary, Canadá
}

\begin{abstract}
RESUMEN
Introducción: Se entiende por lesión catastrófica a cualquier trauma grave que comprometa la cabeza, el cerebro, la columna vertebral o la médula espinal, que pone en riesgo la vida o puede dejar una discapacidad permanente o semipermanente. En la Argentina, la incidencia de lesionados en el ámbito del rugby es alta comparada con la de otros países. En los últimos años, se han implementado múltiples medidas de prevención y se han modificado normas con el objetivo de evitar las lesiones catastróficas. Materiales y Métodos: Se analizaron datos obtenidos de una encuesta telefónica realizada en el marco de colaboración entre la Unión Argentina de Rugby y la Fundación para la Lucha de Enfermedades Neurológicas de la Infancia (Fleni). Se realizó un análisis descriptivo de los datos. Se recopilaron los cambios en las normativas del deporte, que pudieran tener impacto en las futuras lesiones. Resultados: Se observa que el número de lesiones se mantiene estable año tras año. Al asociar este dato con un aumento sostenido de la cantidad de jugadores por año, impresiona haber una disminución relativa del riesgo de lesionarse. Conclusiones: Las lesiones catastróficas generan un gran impacto en la calidad de vida del jugador y de su entorno. Deben considerarse inadmisibles y se deben incrementar los esfuerzos para lograr eliminar los riesgos de lesionarse. El esfuerzo de las entidades reguladoras impresiona tener un impacto positivo al haberse logrado una reducción relativa de las lesiones en relación con el aumento de jugadores año tras año.
\end{abstract}

Palabras clave: Rugby; médula; lesión; catastrófica; scrum.

Nivel de Evidencia: IV

Catastrophic Spinal Cord Injuries in Argentine Rugby. Impact of the Measures Implemented and Their Relative Reduction in Time

\begin{abstract}
Introduction: A catastrophic injury is defined as any serious trauma that involves the head, brain, spine, or spinal cord. They are life-threatening or may leave a permanent or semi-permanent disability. In Argentina, there is a high incidence of injuries. Materials and Methods: Data obtained from a telephone survey carried out in the collaborative framework between the Union Argentina de Rugby and the Fundación para la Lucha de Enfermedades Neurológicas de la Infancia (Fleni, by its acronym) were analyzed. We carried out a qualitative analysis of the data and their relationship to progressive changes in sports regulations. Results: It was observed that the number of injuries remained stable year after year. When associating this fact with a sustained increase in the number of players per year, we can see a relative decrease in the risk of injury. Conclusion: Catastrophic injuries have a great impact on the quality of life of the player and his environment. They must be considered inadmissible and the efforts must be increased to achieve zero risk. In recent years, multiple preventive measures have been implemented and regulations have been modified in order to avoid catastrophic injuries.
\end{abstract}

Keywords: Rugby; spinal cord; scrum.

Level of Evidence: IV

Recibido el 7-7-2020. Aceptado luego de la evaluación el 7-12-2020 • Dr. FEDERICO CARPANI • fcarpani@ gmail.com

https://orcid.org/0000-0003-4696-5994

Cómo citar este artículo: Carpani F, Salvat F, Saco M, Farcy N, Zamorano C, Bruno V, Farez MF, Nogués M. Lesiones medulares catastróficas en el rugby argentino. Impacto de las medidas implementadas y su reducción relativa en el tiempo. Rev Asoc Argent Ortop Traumatol 2021;86(3):335-341. https://doi.org/10.15417/issn.1852-7434.2021.86.3.1154 


\section{INTRODUCCIÓN Y OBJETIVOS}

Durante los últimos 10 años, la profesionalización y la difusión del rugby impulsaron un aumento del número de aficionados en la Argentina; en la actualidad, cuenta con una base de más de 65.000 jugadores.

El rugby es un deporte de equipo en el que hay una fuerte exposición al contacto físico. Tiene situaciones específicas del juego (scrum, tackle, ruck y maul) donde hay una disputa entre dos o más jugadores para determinar la posición de la pelota, y se pueden generar colisiones de alto impacto. Dichas situaciones de juego pueden representar un riesgo de lesiones que pueden tener graves consecuencias. En los últimos 20 años, se ha puesto el foco en los factores de riesgo relacionados con la generación de lesiones graves, y esto llevó a profundizar en la educación y la prevención de estas lesiones.

Se entiende por lesión catastrófica a cualquier trauma grave que comprometa la cabeza, el cerebro, la columna vertebral o la médula espinal, que requiere el ingreso urgente en el hospital y debe resolverse de inmediato, pues plantea un riesgo para la vida o puede dejar una discapacidad permanente o semipermanente. ${ }^{1}$ Estas lesiones representan un porcentaje muy bajo dentro del total de lesiones relacionadas con el rugby, pero, debido a la gravedad y al riesgo potencial de discapacidad, tienden a provocar un impacto significativo en la población general, que percibe el riesgo de forma cualitativa. ${ }^{2}$

La Fundación Unión Argentina de Rugby (FUAR) es una organización no gubernamental sin fines de lucro, fundada en 2015. Su objetivo es proporcionar una asistencia integral a aquellos jugadores que hayan sufrido lesiones graves dentro del campo de juego. Junto con el desarrollo del programa "Rugby Seguro" en 2016, han sido las dos acciones más representativas en todos los aspectos preventivos de las lesiones medulares. Cuentan con una completa base de datos y una importante estructura organizativa centrada en reducir, al mínimo, las trabas generadas por la incapacidad para adquirir una buena calidad de vida.

El objetivo de este artículo es analizar retrospectivamente la historia de las lesiones medulares catastróficas en la Argentina, según los jugadores que la sufrieron, y mencionar las medidas implementadas con el fin de reducir al mínimo estas trágicas lesiones.

\section{MATERIALES Y MÉTODOS}

Dentro del marco de colaboración entre la Unión Argentina de Rugby (UAR) y la Fundación para la Lucha de Enfermedades Neurológicas de la Infancia (Fleni), se buscó actualizar y analizar nuevos datos de los jugadores de rugby que sufrieron lesiones medulares catastróficas entre 1965 y 2017. Para lograr dicho objetivo, se realizó una encuesta telefónica a todos los jugadores incluidos en los registros de la FUAR, que habían sufrido lesiones medulares catastróficas. Miembros de Fleni y CEBES (tanto becarios como médicos) estuvieron a cargo de la comunicación telefónica con los jugadores para realizar una encuesta estandarizada y semiestructurada que permitía la recopilación tanto de datos específicos como de datos subjetivos aportados por cada individuo. Se solicitó el consentimiento verbal de cada jugador para el uso de los datos brindados, pero no se mencionan datos que puedan identificar a los encuestados. Al ser una encuesta personal, se excluyó a los jugadores que habían fallecido antes del estudio. A su vez, se utilizaron los datos estadísticos registrados desde 1996 sobre la cantidad de jugadores incluidos en la UAR.

En la encuesta personal a los jugadores, se obtuvieron datos, como la edad, los parámetros técnicos del juego (posición, horas de entrenamiento) y los detalles de la lesión (situación de juego, detalles biomecánicos de la lesión, ubicación espinal de la lesión). Se registraron la atención médica recibida (en el campo, atención médica, tipo de cirugía requerida, estancia en el hospital) y la evolución crónica (secuelas, reintegración social, relación actual con el rugby). Se decidió evitar el análisis estadístico, ya que, debido a la baja cantidad de jugadores lesionados, no tendría un valor representativo. Se optó por realizar un análisis descriptivo y cualitativo de las lesiones medulares catastróficas. Se recopiló parte de los cambios en las normativas del deporte que pudieran tener impacto en las futuras lesiones.

\section{RESULTADOS}

Se encuestó a 32 de 33 jugadores lesionados entre 1965 y mediados de 2017. El jugador lesionado faltante fue el único que había fallecido por causas relacionadas indirectamente con su lesión medular. El registro fue creado hace 52 años y se han asentado lesiones en 33 jugadores, es decir, un mínimo de un caso por año y un máximo de tres casos por año (2016). 
Todos los jugadores encuestados eran mayores de 18 años cuando se lesionaron. La mediana de edad al lesionarse fue de 19.5 años (rango 15-28). Todos los jugadores lesionados jugaban en forma amateur. El $75 \%$ de los jugadores lesionados realizaba un entrenamiento especializado durante un promedio de $7 \mathrm{~h}$ por semana (entre $2 \mathrm{y}$ 18 h). En cuanto a la posición, 23 de los 32 deportistas jugaban como forwards (74\%), de los cuales 11 ocupaban la posición de Hooker, la posición más riesgosa (Figura 1).

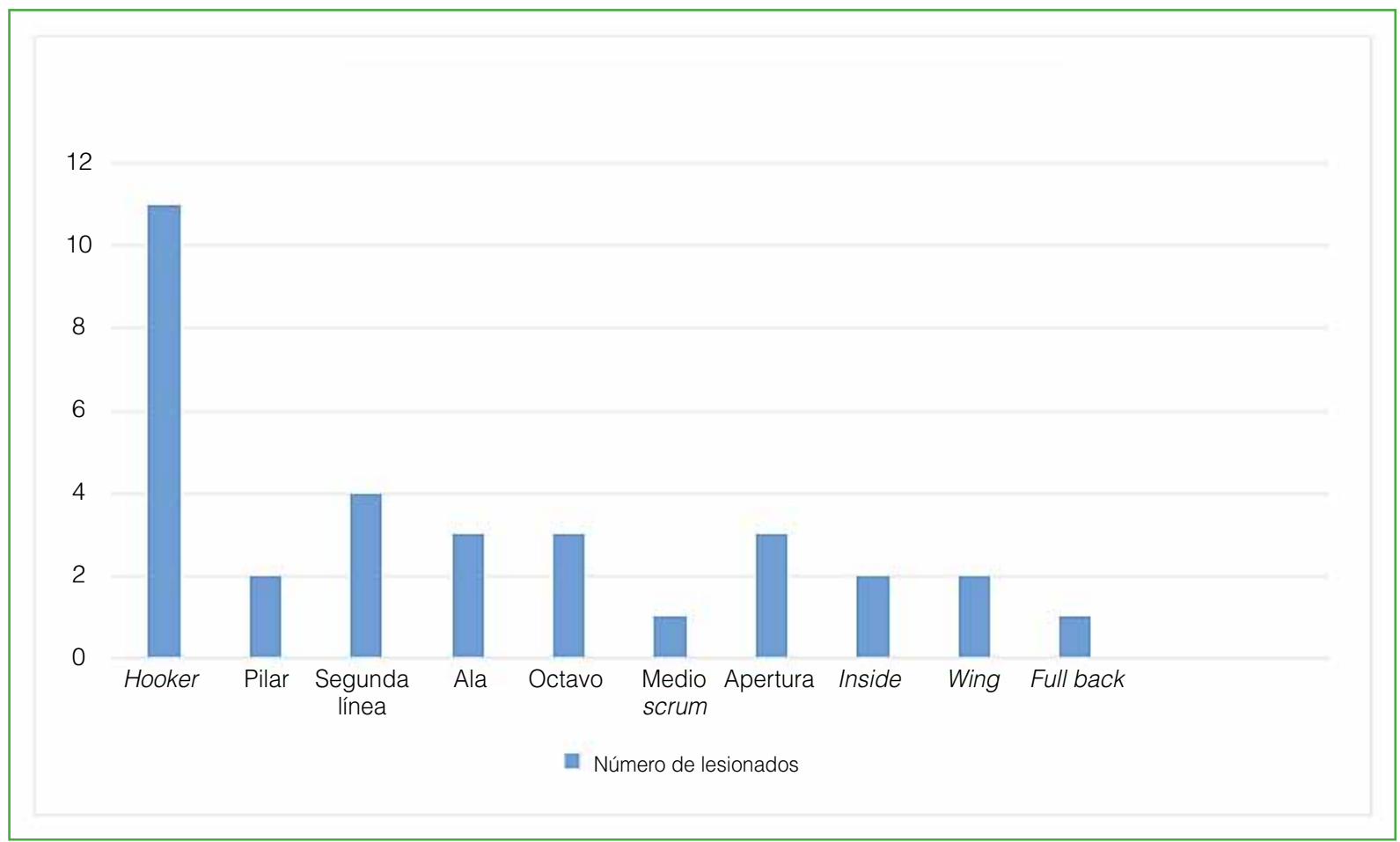

Figura 1. Gráfico de barras que muestra el número de lesionados según la posición. Se destaca la enorme prevalencia de la primera línea de forwards, específicamente la posición de Hooker.

Entre las situaciones del juego, el 50\% de las lesiones se produjeron durante el scrum; el 22\%, en situaciones de tackle; el 19\%, durante el ruck y el $9 \%$ restante, en otras situaciones no especificadas.

Como mecanismo de lesión, se identificó a la hiperflexión cervical con rotación o sin rotación en un 59\% de los casos. El sector de la columna afectado con más frecuencia fue entre los niveles C4-C5 y C6-C7, es posible el compromiso de más de un nivel (Figura 2). Las lesiones de la médula se dividen en sección completa o incompleta, y la primera es la más frecuente.

El 75\% de los jugadores recibió atención médica dentro del campo. El traslado a centros hospitalarios se realizó dentro de los 30 min en el $41 \%$ de los casos, a los 30-60 min en el 40\% y después de 60 min en el resto (19\%). En el $84 \%$ de los casos, se inmovilizó la columna para el traslado del jugador. La mayoría de los lesionados (93\%) requirió cirugía de emergencia para evitar la progresión de la lesión.

Con respecto a la evolución de los jugadores, el 97\% sufrió secuelas motoras que incluyen déficit motor, sensitivo o autonómico. La secuela neurológica más frecuente fue la cuadriparesia con distintos niveles de gravedad. El 87\% logró una adecuada reincorporación al mercado laboral y ese mismo porcentaje permanece vinculado al rugby de alguna manera. Fuera del análisis estadístico, un gran número de los jugadores lesionados opinó que el principal factor por mejorar es el cambio de normas y la educación para la prevención de dichas lesiones. En cuanto a las medidas adoptadas por la UAR, quienes opinaron, consideran que son adecuadas y que se pueden observar cambios en la forma de manejar dicha catástrofe. Su experiencia en primera persona aporta datos útiles que pueden escapar al alcance de los expertos en el tema. 


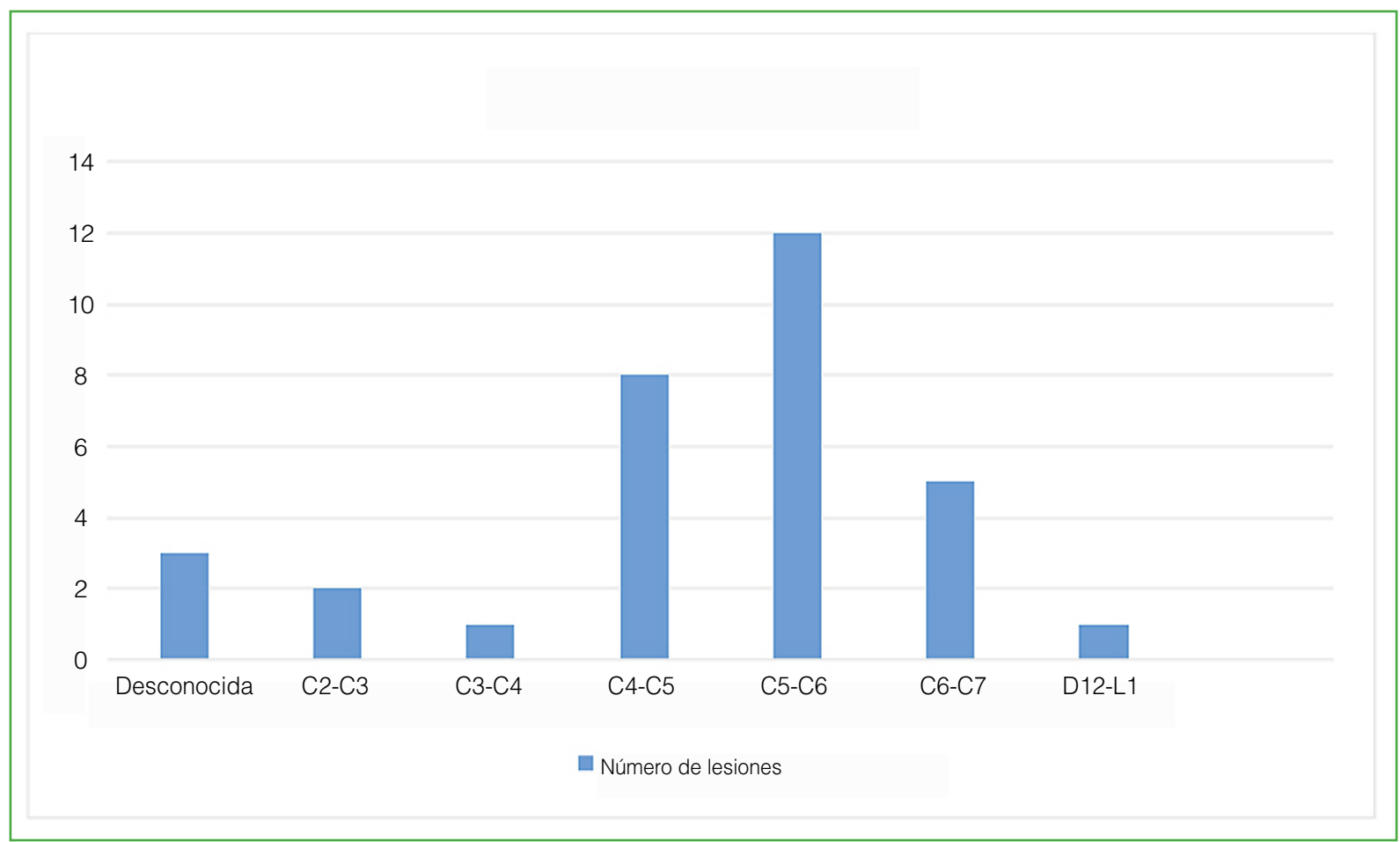

Figura 2. Gráfico de barras que muestra los niveles medulares donde se produjeron las lesiones. Se destaca la prevalencia de la mitad proximal de la columna cervical.

\section{DISCUSIÓN}

Actualmente, en la Argentina, no existen estadísticas oficiales sobre el alcance de las lesiones de la médula espinal asociadas al deporte y no se conoce el impacto económico que estas lesiones generan tanto en la persona como en la sociedad. Chan y cols. llevaron a cabo una revisión de los datos de población proporcionados por cada país. Solo 25 países realizaron estudios epidemiológicos sobre lesiones de columna relacionadas con el deporte, y estas representaron el 13\% de las lesiones medulares. ${ }^{4}$ El rugby se encuentra dentro de un grupo de deportes con un riesgo de moderado a alto de lesiones de la médula espinal. En 1999, Secin y cols. describieron la primera serie de casos de lesiones catastróficas y casi catastróficas en la Argentina. ${ }^{4}$ En el período que analizamos, la incidencia de lesiones fue de 1,4-5,1 casos cada 100.000 jugadores por año. En países con más cantidad de jugadores de rugby, las distintas series reportan la siguiente incidencia de casos cada 100.000 jugadores, por año: Inglaterra 0,48-1,5; Francia 1,4-2,1 y Sudáfrica 1,04-1,8.5,6

Según las estadísticas actuales, se cuenta con más de 65.000 jugadores de rugby afiliados a la UAR. Se observa un crecimiento sostenido de la cantidad de registrados; en los últimos 20 años, se ha duplicado el número de jugadores inscritos en las distintas uniones que dependen de la UAR.

La edad promedio al lesionarse fue de 19.5 años, un momento de transición entre las ligas juveniles y el plantel superior. Los datos bibliográficos son contradictorios con respecto a la relación de la edad con las lesiones catastróficas. No hay series de casos reportados que comparen la dispersión de la edad en las lesiones de la médula espinal dentro de la máxima categoría de edad, ni hay evidencia que respalde que la inmadurez musculoesquelética observada en jugadores más jóvenes o que la diferencia en el estado físico estén relacionadas con la frecuencia de lesiones. ${ }^{6}$ A su vez, en la Argentina, no hay reportes de jugadores profesionales que hayan sufrido este tipo de lesiones, entendiendo que la adecuada técnica y el entrenamiento específico son factores atenuantes de los riesgos en general. ${ }^{5}$

En nuestra serie, cerca de tres cuartos de los lesionados jugaban en posiciones dentro de los forwards. En esta destacada diferencia de lesionados entre los forwards y los backs, los jugadores de la primera línea son quienes están expuestos a un mayor riesgo. En coincidencia con los datos reportados históricamente, la posición de Hooker sobre todo al formar el scrum, continúa siendo la más riesgosa. Estos datos coinciden con las estadísticas 
internacionales y se reflejan en la población argentina. ${ }^{4}$ Como regla general, se tiende a evitar una gran disparidad de formación tanto técnica como física entre los equipos, ya que esto pondría en riesgo a ambas partes. ${ }^{7}$ La recomendación general sigue siendo que se fomente la generación de un marco físico adecuado para el puesto y que se lleve a cabo un entrenamiento específico para los jugadores que participan en el scrum (especialmente las primeras líneas). El scrum es la formación en la que se produce el mayor número de lesiones catastróficas, llegan a la mitad del total entre los jugadores encuestados. En una revisión sistemática publicada en 2015 sobre las lesiones de la columna relacionadas con el scrum, se concluye en que el porcentaje de lesiones de la columna puede ser similar en el tackle y el scrum, pero que las causadas por este último tienden a ser más graves y producen secuelas permanentes. Distintas series han comunicado que las lesiones asociadas al scrum causan un déficit permanente en el $60 \%$ de los lesionados, mientras que las de los tackles provocan un $29 \% .{ }^{8}$ En el scrum es donde mayor cantidad de medidas de prevención se tomaron. Reboursiere y cols. demostraron la eficacia de las normas que conciernen al scrum, implementadas en Francia y cómo disminuyó la incidencia de lesiones medulares. ${ }^{5}$ En la Argentina, se tomaron medidas específicas relacionadas con la prevención de lesiones en el scrum, entre las que se incluyen:

- Modificaciones de las órdenes del árbitro. Actualmente, "Cuclillas - Tomarse - Ya”. Minimizando la embestida entre los bloques de forwards rivales.

- Limitación en las divisiones juveniles de empujar más de 1,5 m cuando se forma un scrum.

- Entrada del árbitro asistente para controlar, junto con el árbitro, ambos lados del scrum.

- Generación de la APL (Acreditación para Primeras Líneas) con capacitación para los jugadores que ocupan esas posiciones.

Se debe tener en cuenta que la gran mayoría de las lesiones graves y no graves se relacionan con el tackle, ya que es la acción individual que más se observa en un partido de rugby. En nuestra experiencia argentina, se identificó que un $22 \%$ de las lesiones se relacionaron con tackles, representaron un porcentaje menor que en otras series. Hay tipos específicos de tackle que aumentan el riesgo de lesionarse (p. ej., tackle alto, más de un "tackleador" o taclear a un jugador que no está de pie), por lo que son considerados ilegales en el rugby moderno. El riesgo de sufrir una lesión medular relacionada con un tackle continúa siendo bajo y las normas establecidas por las entidades reguladoras tienden a disminuir esos riesgos. Año tras año, se refuerza la capacitación a jugadores y a árbitros sobre las adecuadas técnicas y para así evitar lesiones graves.

Con respecto a los mecanismos del trauma, se cree que las lesiones se producen en el momento en el que el jugador sufre una flexión exagerada del cuello, que causa una fractura del muro anterior de la vértebra cervical y una luxación de dicha vértebra hacia el canal medular. ${ }^{8}$ En nuestra serie, este fue el mecanismo reportado como más frecuente. Según la bibliografía, las lesiones con resultados catastróficos se generan en las partes más móviles de la columna cervical, especialmente entre los niveles C4-C5 y C5-C6. ${ }^{9}$ En nuestra serie, el mecanismo de la lesión se identificó como hiperflexión cervical con rotación o sin ella en el 59\% de los casos y más del $90 \%$ fueron entre los niveles C3-C4 y C6-C7. Dicho mecanismo cinético se observó con más frecuencia en el scrum, sobre todo cuando se ven los colapsos de este o cuando un equipo embistió al otro antes de que se haya formado adecuadamente.

La atención médica inicial representa un punto de vital importancia en la evolución de las lesiones catastróficas. Nuestra serie muestra una elevada heterogeneidad en el tipo de tratamiento médico, probablemente relacionado con la dispersión temporal del análisis. En 2017, Badenhorst y cols. informaron una gran variabilidad de la atención médica en diferentes regiones de Sudáfrica y que los niveles socioeconómicos más bajos eran más vulnerables a sufrir estas afecciones, lo que se asoció con un peor pronóstico. ${ }^{10} \mathrm{Si}$ bien, en la actualidad, no se cuenta con una regulación para la atención médica en cancha de los partidos de rugby amateur, se fomenta la capacitación en línea y presencial a los médicos de los clubes y a los que asistan a los partidos dentro de la órbita de la UAR. Las condiciones de transferencia adecuadas y la derivación urgente a centros de alta complejidad en el manejo de patologías neuroquirúrgicas pueden ser la diferencia entre una recuperación completa y secuelas permanentes. Dentro de la órbita del programa BokSmart de Sudáfrica, se desarrolló el programa "Spineline" para facilitar el acceso del jugador lesionado a un centro capacitado para resolver rápidamente la lesión y así evitar su progresión. ${ }^{6}$ En nuestra serie, prácticamente todos los pacientes requirieron algún tipo de instrumentación quirúrgica para lograr la estabilización de la columna cervical y así prevenir la progresión de la lesión de la médula espinal.

El análisis del pronóstico de estas lesiones sigue siendo abrumador. Aunque el 87\% de los pacientes logró obtener cierto grado de reinserción laboral, todos tienen algún tipo de secuelas motoras, sensitivas o autonómicas. El 70\% requiere algún tipo de instrumentación del tracto urinario, lo que genera un grave compromiso de las 
actividades de la vida diaria. Cabe señalar que la mayoría de los jugadores que sufrieron lesiones catastróficas continúan relacionados con el rugby, lo que demuestra la importancia del apoyo emocional y económico brindado por la comunidad del rugby ante este tipo de eventos. Badenhorst y cols. hicieron un interesante aporte al evaluar la calidad de vida de los jugadores de rugby con lesiones medulares en Sudáfrica. Entre las conclusiones, se destaca que el apoyo del grupo deportivo es un factor que mejora la reinserción social de los lesionados. ${ }^{11}$ Con respecto a la opinión de los jugadores lesionados, existe un consenso general de que se está trabajando intensamente en el tema y que las medidas implementadas son adecuadas. Países, como Sudáfrica y Nueva Zelanda, han desarrollado programas para la prevención y el tratamiento de lesiones relacionadas con el rugby (BokSmart y RugbySmart, respectivamente) que requieren la capacitación constante de entrenadores, médicos y árbitros. ${ }^{11,12}$ En la Argentina, el programa "Rugby Seguro" fue diseñado para identificar y retirar a los jugadores que sufren una lesión durante el entrenamiento o el día del partido. Esta iniciativa se lanzó en 2016 con el enfoque principal en la educación de jugadores, el entrenamiento y el personal médico para aumentar el reconocimiento y disminuir la cantidad de lesiones.

Se han implementado tanto programas educativos como numerosos cambios regulatorios para promover la seguridad de los jugadores, por ejemplo,

- Exámenes médicos precompetitivos regulares y la generación de la tarjeta electrónica médica precompetitiva.

- Endurecimiento de las sanciones por juego peligroso, como la tarjeta amarilla agravada.

- Generación de programas de capacitación específica para jugadores, autoridades y médicos.

• Fomentar la premisa de "IDENTIFICAR Y RETIRAR" ante la sospecha de una lesión importante.

Estos cambios han fortalecido la idea del juego seguro y muestran sus resultados a corto plazo, ya que se ha logrado mantener un bajo número de lesiones de la médula espinal, pese a un aumento significativo de la cantidad de jugadores. Esto se puede interpretar como una disminución relativa de las lesiones catastróficas a medida que aumenta el número de jugadores cada año. Esta disminución relativa en el riesgo anual de lesiones catastróficas se asocia con la intensificación y promoción de los programas de educación continua combinados con regulaciones menos flexibles. A su vez, el resultado de estos cambios se tradujo en la ausencia de nuevas lesiones catastróficas desde 2017 hasta la fecha.

Nuestro estudio tiene ciertas limitaciones, como la extensión temporal del registro, cuando las reglas han cambiado en múltiples ocasiones y hay una transición del rugby amateur al profesional. A su vez, el seguimiento a largo plazo y la dificultad para nuclear a todos los lesionados pueden generar que no todos los pacientes hayan sido incluidos en la encuesta. La incidencia calculada corresponde a la cantidad de jugadores lesionados por la cantidad de jugadores por año, al no tratarse del riesgo según horas de exposición, los resultados pueden ser inexactos. Creemos que este debería ser el punto de partida para un análisis prospectivo sobre el impacto de las medidas de prevención, y el inicio de trabajos colaborativos y multidisciplinarios que reduzcan este tipo de lesiones a la mínima expresión, mientras se mantiene intacto el espíritu del rugby.

\section{CONCLUSIONES}

Durante las últimas cuatro décadas, la cantidad de pacientes con lesiones medulares relacionadas con el rugby en la Argentina permaneció estable. Al compararla con el aumento del número total de jugadores, se logra una disminución relativa en la relación entre jugadores lesionados y el total de jugadores cada año. Así se demuestra que los esfuerzos para mejorar las reglas y la educación constante son un factor fundamental para la prevención. El tackle y el scrum son las situaciones del juego más riesgosas y en las que se ha puesto más énfasis para reducir, al mínimo, los riesgos de sufrir este tipo de lesión. Especialmente las primeras líneas de todas las divisiones deben recibir educación teórica y práctica supervisada para lograr una técnica adecuada que disminuya los riesgos. Es fundamental profundizar el aprendizaje de las técnicas correctas de abordaje en las formaciones, especialmente en el ámbito de las divisiones juveniles.

Conflicto de intereses: Los autores no declaran conflictos de intereses. 
ORCID de F. Salvat: https://orcid.org/0000-0003-1479-2312 ORCID de M. Saco: https://orcid.org/0000-0002-4248-8361

ORCID de N. Farcy: https://orcid.org/0000-0002-8044-516X

ORCID de C. Zamorano: https://orcid.org/0000-0002-4902-7189
ORCID de V. Bruno: https://orcid.org/0000-0003-2378-6328

ORCID de M. F. Farez: https://orcid.org/0000-0002-9754-8771

ORCID de M. Nogués: https://orcid.org/0000-0003-4319-6456

\section{BIBLIOGRAFÍA}

1. Wolff CS, Cantu RC, Kucera KL. Catastrophic neurologic injuries in sport. Handb Clin Neurol 2018;158:25-37. https://doi.org/10.1016/b978-0-444-63954-7.00004-5

2. Fuller CW. Catastrophic injury in rugby union: is the level of risk acceptable? Sports Med 2008;38(12):975-86. https://doi.org/10.2165/00007256-200838120-00002

3. Chan CW, Eng JJ, Tator CH, Krassioukov A. Epidemiology of sport-related spinal cord injuries: A systematic review. J Spinal Cord Med 2016;39(3):255-64. https://doi.org/10.1080/10790268.2016.1138601

4. Secin FP, Poggi EJ, Luzuriaga F, Laffaye HA. Disabling injuries of the cervical spine in Argentine rugby over the last 20 years. Brit J Sports Med 1999;33(1):33-6. https://doi.org/10.1136/bjsm.33.1.33

5. Reboursiere E, Bohu Y, Retière D, Sesboüé B, Pineau V, Colonna JP, et al. Impact of the national prevention policy and scrum law changes on the incidence of rugby-related catastrophic cervical spine injuries in French Rugby Union. Brit J Sports Med 2016;52(10):674-7. https://doi.org/10.1136/bjsports-2016-096122

6. Badenhorst M, Verhagen E, Lambert M, Mechelen WV, Brown J. When this happens, you want the best care: Players' experiences of barriers and facilitators of the immediate management of rugby-related acute spinal cord injury. Qual Health Res 2019;29(13):1862-76. https://doi.org/10.1177/1049732319834930

7. Quarrie KL, Cantu RC, Chalmers DJ. Rugby union injuries to the cervical spine and spinal cord. Sports Med 2002;32(10):633-53. https://doi.org/10.2165/00007256-200232100-00003

8. Trewartha G, Preatoni E, England ME, Stokes KA. Injury and biomechanical perspectives on the rugby scrum: a review of the literature. Brit J Sports Med 2014;49(7):425-33. https://doi.org/10.1136/bjsports-2013-092972

9. Fiorillo P, Demonti H, Giuria H, Aparicio JL, Della Vedova F. Trauma deportivo cervical catastrófico en un jugador de rugby. Rev Asoc Argent Traumatol Dep 2014:21 (1). Disponible en: https://g-se.com/trauma-deportivo-cervicalcatastrofico-en-un-jugador-de-rugby-1821-sa-H57cfb27254339

10. Badenhorst M, Verhagen EA, Mechelen WV, Lambert MI, Viljoen W, Readhead C, et al. A comparison of catastrophic injury incidence rates by Provincial Rugby Union in South Africa. J Sci Med Sport 2017;20(7):643-7. https://doi.org/10.1016/j.jsams.2017.01.232

11. Badenhorst M, Brown JC, Lambert MI, Mechelen WV, Verhagen E. Quality of life among individuals with rugbyrelated spinal cord injuries in South Africa: a descriptive cross-sectional study. BMJ Open 2018;8(6):e020890. https://doi.org/10.1136/bmjopen-2017-020890

12. Quarrie KL, Gianotti SM, Hopkins WG, Hume PA. Effect of nationwide injury prevention programme on serious spinal injuries in New Zealand rugby union: ecological study. BMJ 2007;334(7604):1150.

https://doi.org/10.1136/bmj.39185.605914.ae 